Voix et Images

voixetimages

\title{
Quête d'un discours fondateur chez Jean-Claude Germain
}

\section{Inês Signorini}

Volume 13, numéro 2 (38), hiver 1988

Le propre du corps Roger Des Roches

URI : https://id.erudit.org/iderudit/200712ar

DOI : https://doi.org/10.7202/200712ar

Aller au sommaire du numéro

Éditeur(s)

Université du Québec à Montréal

ISSN

0318-9201 (imprimé)

1705-933X (numérique)

Découvrir la revue

Citer cet article

Signorini, I. (1988). Quête d'un discours fondateur chez Jean-Claude Germain.

Voix et Images, 13(2), 304-316. https://doi.org/10.7202/200712ar d'utilisation que vous pouvez consulter en ligne.

https://apropos.erudit.org/fr/usagers/politique-dutilisation/ 


\section{Quête d'un discours fondateur chez Jean-Claude Germain}

\section{par Inês Signorini, Universidade Federal da Paraíba, Brésil}

La vie n'est que l'opacité du rêve: c'est un comme si dont toute l'originalité relative tient dans la surimpression d'une suite décousue de comparaisons énigmatiques. En somme, les serrures n'existeraient pas qu'il faudrait tout de même s'inventer des clés, pour caresser les éléphants.

(Jean-Claude Germain, l'École des rêves)

Mais toute la question est précisément de savoir si la philosophie [...] et la littérature aussi bien n'ont pas toujours affaire à des éléphants et à des tortues - en d'autres termes, si l'aveu d'ignorance (ou ce qui revient au même, la fiction du manuscrit illisible) n'est pas au même titre que la positive assurance de savoir, une ruse déployée en vue de se donner un cran d'arrêt.

(L. Dallenbach, le Récit spéculaire)

En 1979, après plus de dix années de «présence» dans le milieu thêâtral québécois, Jean-Claude Germain parlait de l'existence d'un conflit entre l'auteur et l'acteur à l'intérieur de la société. Tout en ayant autorisé une «redéfinition» de ces deux rôles, la création collective de la fin des années 60 n'avait pas, selon lui, éliminé le problème 1 . La primauté accordée à l'auteur dans la période suivante, précisait-il plus tard, relevait d'une redistribution du pouvoir créateur entraînée justement par cette redéfinition des rôles, et cela en accordance parfaite avec l'évolution de la société québécoise. Ainsi, la réapparition de l'auteur dans le travail théâtral était légitimée, à son avis, aussi bien par le statut de la fonction d'écrivain dans l'économie du spectacle - le maître des situations dramatiques et des mots ${ }^{2}$ - que par le statut du rôle de ce «maître» dans la mécanique de fonctionnement d'un collectif. Ce collectif devait comprendre les différents

1 Jean-Claude Germain, «l'Acteur, l'improvisation, la création collective», Jeu, 13 , automne 1979 , p. 51-57.

2 «Jean-Claude Germain: au théâtre d'aujourd'hui», entretien/Bernard Andrès et Yves Lacroix, Voix \& images, vol. VI, $\mathrm{n}^{\circ} 2$, hiver 1981, p. 183. 
créateurs participant à la construction du spectacle, mais aussi les individus participant à la construction du Québec ${ }^{3}$. À la fonction d'auteur dramatique correspond donc le rôle social d'un acteur collectif - celui qui parle au nom et pour les autres, selon la formule du dramaturge.

Néanmoins, cette fonction d'auteur dramatique tend ici à intégrer celle de metteur en scène dans la mesure où «l'organisateur du jeu» se veut un écrivain scénique ${ }^{4}$ : c'est-à-dire un créateur/ «artisan» et un auteur de littérature thêâtrale. Il s'agit avant tout d'aboutir à une écriture scéniquement efficace mais tout aussi définitive (dans le sens mallarméen du «texte incorruptible» en vue du «spectacle futur»). L'écriture dramatique fonctionnera donc comme une partition, la plus exacte, la plus explicite pour que d'autres artisans puissent fidèlement la reproduire dans l'avenir 5 . Cette partition originale rejoint le projet d'un discours culturel à la hauteur d'un pays et non pas d'une province, ou d'une copie, ou d'un souvenir. Il s'agit de «traduire» culturellement la modernité d'un pays resté folklorique par la force des choses ${ }^{6}$. Le caractère totalisateur du projet ne fait pas de doute: la partition sera jouée par tous ensemble à un moment ou à un autre, comme l'avait suggéré, déjà en 1974, Michel Bélair7.

À ce propos, dans l'ensemble des créations de Germain ${ }^{8}$ ainsi que dans ses textes explicatifs et publicitaires, deux aspects de sa démarche paraissent particulièrement révélateurs de la progression de son travail théâtral et de sa réflexion critique. Ces deux aspects se recoupent depuis les premières productions, mais ne se confondent qu'après bien des années de pratique théâtrale et de réflexion sur la «réalité» du Québec.

Le premier volet se rapporte au développement thématique - sur quoi parler - et aux formes correspondantes de théâtralisation - comment parler. Cette recherche de l'authentique, par opposition au conventionnel aliéné et aliénant, allait présider au choix des thèmes et des moyens de construction dramaturgique.

Ainsi, tout en posant la question globale de la structure familiale rétrogade Germain mettait en place des ressorts dramatiques empruntés à la tradition

3 Ibid.

$4 \quad$ Ibid., p. 177.

5 Ibid, p. 176.

6 Jean-Claude Germain, "Quel thêâtre populaire», Jeu 13, automne 1979, p. 62.

7 «Jean-Claude Germain: "Sarah Ménard, c'est tout ce que nous sommes"», le Jour, 9 novembre 1974, p. 17.

8 En fonction cependant de l'importance du texte écrit comme «aboutissement» - selon l'expression de Germain lui-même - de son travail d'écriture scénique, nos références de base ont été empruntées plutôt aux pièces dont les textes ont été publiés. En fait, ces partitions fonctionnent comme des cristallisations des thèmes et des techniques développés ou à peine esquissés dans les pièces et spectacles inédits. Le caractère sélectif de ce travail d'auteur le rend particulièrement révélateur de la démarche personnelle de Germain. 
québécoise de la revue et des amusements populaires. Les conventions théâtrales étant plutôt étrangères aux habitudes culturelles du grand public québécois, il fallait, d'après Germain, traduire «théâtre» par «spectacle»9. Cette reprise d'une tradition dite «marginale» car véhiculée par une culture populaire «pré-historique» était donc assimilée à la question de l'authenticité du langage. Le public «populaire» restait cependant, à cette époque, une notion plutôt vague: $J^{\prime}$ entends par «populaire» un théâtre que tous comprennent, c'est-à-dire la grande majorité des Québécois. 10

Ce populisme récurrent dans les interventions du dramaturge nous renvoie au deuxième volet de sa démarche, auquel nous avons fait allusion ci-haut: sur le rôle attribué au public, et sur le rapport envisagé entre la communauté et les «gens de thêtre», entre la scène et la salle.

Germain s'expliquait ainsi en 1973:

Il y a une chose que je n'ai jamais faite et que je ne ferai jamais. Je n'ai jamais fait de spectacle POUR le public ou un certain public, ou encore selon une certaine idée que j'avais du public. J'ai fait des spectacles pour exprimer, pour que nous nous exprimions, nous, les P'tits Enfants Laliberté, ce que nous avions à dire. Il s'est avéré que nous avions vu juste, particulièrement auprès des jeunes. 11

Il faut rattacher cette négation du destinataire, au moment de la création, au refus du public conventionnel, pris en même temps comme thème et comme cible dans les pièces destinées à «liquider» l'univers socio-culturel et politique dominant. Diguidi diguidi ha! ha! ha!12 illustre bien les propos de cette période.

Quelques années plus tard, lors de la création de l'École des rêves en 1978, la question du rapport avec le public était envisagée d'un autre point de vue:

Dix ans de pratique théâtrale ont fait du public, chez moi, le souci omniprésent. C'est lui qui corrige ou impose ses corrections au spectacle, c'est à cause de lui que la critique est incarnée. Le Créateur se doit

9 Il y a ici une culture globale du spectacle beaucoup plus qu'une culture de théâtre spécifique [...] Le mot Théâtre a conditionné un certain nombre d'attitudes qu'on a voulu éliminer pour créer un contact plus chaleureux et plus naturel entre ce qui se passe sur la scène et ce que les gens peuvent percevoir dans la salle (Albert Brie, «Jean-Claude Germain au bord d'un Affront commun», le Devoir, 3 novembre 1973, p. 19.

10 Ibid.

11 Ibid. Souligné par l'auteur.

12 Jean-Claude Germain, Diguidl, diguidi, ha! ha! ha!, Montréal, Leméac, coll. «Théâtre canadien», $\mathrm{n}^{\circ} 24,1972,194 \mathrm{p}$. 


\section{de chercher de nouvelles techniques de racontement. 13}

Ce «souci» du public se traduisait alors par un souci de commmunication avec le plus grand nombre - une communication en trois dimensions, à échelle humaine. Le public-cible étant mieux défini - les classes moyennes - le «populaire» était dès lors confondu avec le spectaculaire associé à des soins de production chaque fois plus rigoureux. Cette tentative de «brancher» la salle sur une «partition commune» par le rire - un bon rire du ventre, de tous les ventres riant à l'unisson 14 _, et aussi par l'étonnement et l'émerveillement, a débouché sur la conception du spectacle célébration: d'un côté le rituel d'exorcisme collectif (A canadian play/Une plaie canadienne15) et de l'autre le show «like a year-end holiday celebration», selon l'expression de Maureen Peterson 16 (les Nuits de I'Indiva ${ }^{17}$ ).

Avec la création de ces deux spectacles, l'authenticité des thèmes et des formes de thêâtralisation se confondait avec celle du rapport avec le public. Au fil des années, cette quête, qui se traduisait au début par la «liquidation» d'un Québec authentique mais rétrogade (le pays des «assis» de Diguidi diguidi ha! ha! ha!), est passée par le recensement des biens collectifs (l'héritage des Sansoucis, la récupération du folklore national, l'arrière-pays des comédiens ambulants et la culture «gastronomique» des villes) pour aboutir à la prise de conscience de soi par l'acceptation consciente de ses racines (la diva «kétaine» et «ridicule»). Le mouvement est circulaire: on part de la réalité d'un pays oublié ou ignoré et on y retourne comme seul moyen de ne pas s'oublier, de ne pas se perdre pour de bon, (comme disait Épisode Surprenant). Au souci initial d'expression d'une altérité et par conséquent d'une distance insurmontable par rapport au public moyen, a suivi l'affirmation chaque fois plus véhémente d'une «substance» commune à exalter jusqu'au paroxysme. Le rapport de complémentarité entre la scène et la salle devait se consolider d'une manière sensible, d'où l'analogie avec l'orgasme, déjà établie par la diva dans son premier spectacle. Retrouver l'authentique équivaut ainsi à retrouver l'immédiat, la présence et la propriété à soi par la communication plénière, selon la perspective rousseauiste de la «parole transitive», développée par la tradition philosophique du sujet 18 . Dans la perspective mallar-

13 Adrien Gruslin, «Jean-Claude Germain: le rire contre la bêtise», le Devoir, 8 avril 1978 , p. 49.

14 Martine Corrivault, «le Théâtre, comme tout ce qui doit durer, ça ne s'improvise pas par n'importe qui», le Solell, 27 mars 1976, p. D2.

15 Jean-Claude Germain, A canadian play/Une plaie canadienne, Montréal, VLB éditeur, 1983, 222 p.

16 «La Nuit packs a barrell of laugh». The Gazette, 12 janvier 1980, p. 72.

17 Jean-Claude Germain, les Nuits de l'Indiva, Montréal, VLB éditeur, 1983, $155 \mathrm{p}$.

18 Jean Starobinski, Jean-Jacques Rousseau. La transparence et l'obstacle, suivi de «Sept essais sur Rousseau», Paris, Gallimard, 1971, p. 320-429.

Jacques Derrida, «la Structure, le signe et le jeu dans le discours des sciences humaines», l'Écriture et la différance, Paris, Seuil, 1967, p. 409-428. 
méenne du «spectacle futur», à la célébration et au «texte incorruptible», il faut ajouter la foule dépositaire du «mystère». C'est le type d'expérience culturelle recherché par Germain ${ }^{19}$. Passer du public-cible de la première étape (les «assis» et les impuissants du présent) à une référence beaucoup plus élargie - souvent virtuelle et fantasmatique, selon l'expression du dramaturge -, source et miroir des images et des rêves projetés par le théâtre spectacle: le pays qui sera ce qu'il est déjà, celui de l'avenir.

Cette idée de faire surgir le pays qui est dans le pays entraîne un double mouvement vers le passé - retour au pays oublié, refoulé, enfoui dans tous et dans chacun - et vers l'avenir - projection du rêve commun.

\section{Vers le passé: le discours historique}

Lors de la création de Un pays dont la devise est je m'oublie 20 en 1976, Germain justifiait le titre et le sujet de sa pièce par son goût du jeu de mots - il s'était déjà défini comme peintre calembourgeois du dimanche 21 . Il invoquait aussi son goût de l'histoire. Une histoire si mal écrite, selon lui, qu'il fallait remettre les choses dans leur juste perspective 22 .

En fait, il serait hasardeux de vouloir dissocier son intérêt pour l'histoire y compris la légende, l'anecdote et la tradition religieuse - de son goût du calembour et de toutes sortes de jongleries avec le langage. Et cela parce que les jeux de l'ironie, de la parodie, les variations si particulières sur des thèmes communs, si nombreux dans ses pièces et dont l'effet de bariolage affecte l'ensemble du spectacle, témoignent de ce même souci de remettre les choses dans leur juste perspective.

Comme l'a bien signalé Laurent Mailhot, l'histoire qui fait l'objet de la réflexion de Germain, ainsi que d'autres dramaturges québécois, est en réalité une galerie d'espaces narratifs où des éclairages, des décors, des costumes, des gestes (isolés) [...] masquent la vue et l'action d'ensemble ${ }^{23}$. Il s'agit donc d'un récit multiple et décousu, d'un discours troué, réunissant plutôt des masques et des fragments - des scènes de théâtre, comme le veut Mailhot. Pour rétablir la juste perspective des faits historiques, Germain a fait appel aussi bien à l'historiographie traditionnelle (celle des archives) qu'aux théories psychanalytiques susceptibles d'expliquer l'inexplicable (capables d'ajouter une dimension nouvelle aux

19 Jean-Claude Germain, «le Théâtre d'amateurs», Jeu, 13, automne 1979, p. 64.

20 Jean-Claude Germain, Un pays dont la devise est je m'oublie, Montréal, VLB, éditeur, 1976, $138 \mathrm{p}$.

21 Adrien Gruslin, «Jean-Claude Germain... dérision, exorcisme, humour», le Devoir, 19 juillet 1975, p. 9.

22 Joseph Rudel-Tessier, «J.-C. Germain raconte l'histoire des Québécois», la Presse, 27 mars 1976, p. D7.

23 «De l'actualité à l'histoire (ou l'inverse)», Théâtre québécois II. Nouveaux auteurs, autres spectacles, Montréal, Hurtubise HMH, 1980, p. 33. 
notions traditionnelles de causalité et de réalité). Ainsi, la théorie freudienne de la trace mnésique, l'idée de l'histoire comme «récit-fiction» du refoulé 24 , outre les études psychanalytiques sur les rêves et les hypothèses lacaniennes sur la structure discursive de l'inconscient en tant qu'espace-temps du désir ${ }^{25}$, ont fourni au dramaturge l'essentiel de l'outillage destiné à rétablir une raison, là où il ne voyait que rupture et perte du sens.

Cette mise en perspective de l'histoire et des mythologies anciennes en fonction des interrogations actuelles a débouché chez Germain sur la constatation d'une opacité absolue des signes (des mots et des images). À cause des agents d'oppression (les Anglais, les curés, les politiciens de la petite tabbe) mais aussi de la dyslexie caractérielle propre à «l'homo quebecensis» d'hier et d'aujourd'hui26. L'état d'aliénation se traduit ainsi par une incapacité individuelle et collective de saisir aussi bien le sens des récits que celui des faits. On n'a donc prise ni sur le langage figé de la tradition et des versions officielles des faits anciens et nouveaux, ni sur la réalité non médiatisée par le discours. Seul un tel handicap expliquerait, selon le dramaturge, l'imbroglio psychotique dont témoigne la plupart des choix collectifs des Québécois, depuis le temps de la colonisation jusqu'à nos jours. Le concept de la souveraineté-association qui a débouché sur le résultat de la consultation référendaire de 1980 illustre bien, selon lui, cette particularité québécoise, capable de transformer l'échec du colonisateur en une catastrophe.

Mais cette difficulté à lire le Québec se rattache aussi bien au caractère spéculaire de l'histoire officielle, où se déploie une multiplication incontrôlée de représentations et de toutes sortes de reflets. Le bilinguisme serait, selon Germain, la principale cause de cette reproduction spéculaire, car toute traduction est une trahison, productrice de malentendus et de quiproquos. Le principe psychanalytique de l'impossibilité d'une traduction sans déperdition, notamment lorsqu'il s'agit du langage onirique, a renforcé ce thème, souvent repris chez Germain depuis Si les Sansoucis [... $]^{27}$. La question n'a jamais été mieux explicitée dans ses implications historiques que dans A canadian play/Une plaie canadienne, comme le suggère le titre même de la pièce.

Ayant identifié les principaux facteurs d'obscurcissement du discours originaire, Germain s'est donc lancé dans le déchiffrement et la reconstruction du sens dans le récit historique. Il s'agissait alors d'articuler les mots et les choses, de façon à rétablir une certaine lisibilité dans le récit des événements passés et dans

24 Michel de Certeau, «la Fiction de l'histoire», l'Écriture de l'histoire, Paris, Gallimard, 1975, p. 312-358.

25 Jacques Lacan, «l'Instance de la lettre dans l'inconscient», Ecrits, Paris, Seuil, 1966, p. 439-528.

26 «Le Cabinet de réflexion», A canadian play/Une plale canadienne, p. 23.

27 Jean-Claude Germain, Si les Sansoucis s'en soucient, ces Sansoucis-ci s'en soucieront-ils? Bien parler, c'est se respecter!, Montréal, Leméac, coll. «Théâtre canadien», n²4, 1972, p. 99. 188. 
l'approche des faits contemporains.

Suivant la même perspective, il fallait retrouver un fil conducteur, un principe de groupement des discontinuités. La découverte, pourrait-on dire, de Lord Durham et de son rapport instituant la politique d'assimilation a permis justement de retrouver ce centre, cette perspective autour de laquelle pouvaient se rattacher de façon conséquente des récits et des représentations apparement disparates ou contradictoires. Germain a ainsi justifié l'importance dudit rapport:

[...] une étude de trois cents pages qui encore aujourd'hui demeure un document d'une lucidité implacable. D'autant plus remarquable qu'il annonçait la conquête du gouvernement responsable et la naissance du Commonwealth britannique en même temps qu'il laissait entrevoir la Confédération et fixait la stratégie du dialogue et de la collaboration entre Canadiens français et Canadiens anglais qu'on allait suivre pendant plus d'un siècle 28 .

Une fois retrouvée cette parole non déguisée, ce deuxième discours originaire - point de départ de la Grande Réponse à Lord Durham que d'aucuns nomment l'Histoire du Canada 29 - il fallait cependant retrouver le sujet du discours, remonter cette parole à son entité productrice. Il fallait incarner le personnage. Dans ce sens, l'homme Durham est aussi important que son discours, car il incarne au niveau de l'image - le personnage, «le bonhomme» - et au niveau ontologique - le «père» mythique ${ }^{30}$ - un point d'ancrage, une sorte de conscience ordonnatrice de l'histoire. À ce propos, il est intéressant de voir comment Germain le met en scène: Aujourd'hui, je vois Durham assis au fond des temps 31 .

Au niveau dramatique, cette conception du «Bonhomme» en tant que présence consciente et verbe créateur a permis une remise en perspective de la galerie des tableaux historiques québécois. La création de Un pays dont la devise est je m'oublie est entièrement orientée vers cette réécriture de l'histoire, où la redistribution des rôles a conféré aux héros traditionnels le statut de masques vides ou de «cabotins» ridicules (le découvreur, l'intendant et le général français) et aux «apocryphes» traditionnels le statut de héros attendant leur tour pour rentrer dans l'histoire (le coureur des bois, le Canayen, l'habitant). Suivant un nouveau découpage, l'histoire moderne inaugurée par «l'Émeute du Forum», devient celle des représentations incarnées par les héros de la mythologie populaire, comme Louis Cyr et Maurice Richard.

Cependant, pour que ces héros de légende deviennent des héros de l'histoire,

28 «Le Bonhomme», A canadian play/Une plaie canadienne, p. 198.

29 Un pays dont la devise est je m'oublie, p. 10.

30 «Le Cabinet de réflexion», p. 24.

31 Adrien Gruslin, «la Filière canadienne selon Germain», le Devoir, 28 avril 1979, p. 29. 
ou plutôt pour que le pays prenne conscience de soi, il fallait, selon Germain, que soit exorcisée la catastrophe historique, la «chute», représentée par la Conquête dans ses trois versions (la Conquête proprement dite, la Défaite et la Confédération). Aussi, dans son rituel d'exorcisme collectif - A canadian play/Une plaie canadienne - a-t-il essayé de racheter d'une part la dispersion de la conscience collective instaurée par l'arrivée des Anglais et d'autre part la dispersion de la conscience individuelle, prise au vertige d'un univers spéculaire - cette chasse-galerie de doubles malfaisants et d'ombres maléfiques $q u$ 'a engendrée notre berlue collective 32 . Lord Durham s'est retrouvé au centre du rituel sacrificiel, dont la référence maçonnique apparente, ainsi que la référence psychanalytique très prononcée, n'effacent pas complètement la référence judéochrétienne.

En effet, au «Bonhomme» est accordé le statut d'un dieu/auteur (conscience créatrice, cohérence et lucidité) et, à un autre niveau, celui d'un homme/dieu ou d'un dieu humanisé dans le sens biblique, c'est-à-dire capable d'assurer le salut - ou le châtiment - individuel et collectif. Selon la perspective freudienne de la conscience malheureuse en tant que conscience de la séparation, à la mort du premier (le meurtre du père) s'associe la liquidation du deuxième, cet innocent/coupable, ce bouc émissaire conscient de son rôle (le sacrifice judéochrétien).

De même, le discours du «Bonhomme» - celui de la mémoire historique fonctionne comme un contrepoint du discours religieux sur les origines de la québécité - celui de la tradition officielle, ou la tradition de l'oubli et de la démission collective, selon les formules du dramaturge. À la franc-maçonnerie (double laïcisé de l'institution religieuse) sont associés des schèmes formels et conceptuels renvoyant plutôt à la métaphysique qu'à la psychanalyse, ce qui renforce la référence au modèle religieux, perçu en négatif.

Conçue après les élections du 15 novembre 1976 et à la veille de la consultation référendaire de 1980 , cette pièce représente l'aboutissement d'une étape - celle de l'appropriation de l'histoire par la parole et par l'image de l'Autre - et cela en vue d'une nouvelle étape tournée davantage vers le présent et l'avenir.

\section{Vers l'avenir/présent: le rêve commun}

L'idée d'un rêve commun pour donner un sens québécois au Québec et ainsi le délivrer du modèle canadien d'un deal entre une gang de gérants de banque relève chez Germain d'une conception du pays comme entité culturelle distincte, particulière, autonome et souveraine ${ }^{33}$. Ce genre de mystère ${ }^{34}$ se manifeste dans

32 «Le Cabinet de réflexion», p. 13.

33 Jean-Claude Germain, «Un pays, c'est avant tout un rêve commun», le Pays théâtral, saison 1977-1978, vol. $1, n^{\circ} 2$, p. 4.

34 Jean-Claude Germain, «l'État et la culture», Jeu, 13, automne 1979, p. 70. 
l'acte créateur. Le grand obstacle à la manifestation de cette réalité qui s'ignore - le Québec n'a toujours été rien d'autre qu'une réalitée 35 - serait la dispersion/aliénation des consciences et, par conséquent, la décomposition de l'image de soi au niveau individuel et collectif.

Ainsi, son appel à l'élaboration d'un rêve collectif, une sorte d'image régulatrice de la québécité en devenir, s'est-il toujours fait dans le sens d'une création collective, fondée par l'avènement d'un geste et d'une parole originaires.

Suivant la perspective psychanalytique du rêve comme langage de l'imaginaire, comme accès à la subjectivité la plus intime, Germain a tenté de faire de son thêatre un appel puissant au rêve et en même temps un moment privilégié de manifestation de cette évidence culturelle et ontologique qui fait, d'après lui, la différence entre la vie et la survivance - la survivance n'est pas le fait des créateurs: c'est le lot des conservateurs 36.

Renouant aussi avec le discours sociologique des années 70 sur les rapports entre fête et libération 37 , Germain a fait de la danse l'allégorie la plus éloquente de ce fond ontologique qui fait de chacun un pays: mononcque Simon, le corps ben drette qui jiguait du haut de ses six pieds... (...) Stait la forêt en marche... stait lpays... Stait lpays dboutte qui dansait! 38

Cette image, associée aussi à celle du yable ou l'bon dieu Pan est à la source du talent et de la folie de sa diva et de tous les possédés, c'est-à-dire ceux qui, selon la conception du dramaturge, ont atteint ce centre inamovible, le but et la pierre philosophale des créateurs: la présence réelle 39 .

Mais dans cette retraite vers le «centre», vers la possession de soi, Germain tend à se retourner vers un espace-temps originaire, aux frontières de la nature et de la civilisation, du geste et de la parole, de la mémoire et de l'oubli. C'est l'espace-temps de la gigue de l'oncle Simon, de la chanson de la Maumariée, de l'errance et du libertinage du coureur de bois; c'est l'espace-temps d'avant le bien et le mal, d'avant la «chute» 40 .

35 Adrien Gruslin, «la Filière canadienne selon Germain», op. cit.

36 Jean-Claude Germain, «Un pays, c'est avant tout un rêve commun», op. cit.

37 Alfred Simon, «l'Idéologie de la fête», les Signes et les songes. Essai sur le théâtre et la fête, Paris, Seuil, 1976, p. 119-169.

38 Jean-Claude Germain, les Hauts et les bas d'la vie d'une diva: Sarah Ménard par eux-mêmes, Montréal, VLB éditeur, 1976, p. 141.

39 Jean-Claude Germain, «Ernest Guimond 1897-1977 un grand comédien dans une petite époque», le Pays théâtral, saison 1977-1978, vol. $1, \mathrm{n}^{\circ} 1$, p. 4.

40 J'ai lpied gauche dan-z-un monde pis lpied drette dans l'autte! Chus squ'y z-appellent icitte un homme libbe! [...] J'ai pas d'attaches, chus comme un radeau à la dérive, jsuis l'courant! Chcroué ni a dieu, ni a diabbe! Mais si on pouvait s'inventer un arc-en-ciel, j'y croirais ptête! (Jean-Claude Germain in Mamours et conjugat. Scènes de la vie amoureuse québécoise, 
Tous les archétypes viennent se greffer sur cette figure d'un temps d'avant le temps, à la Rousseau, d'un temps du rêve à plein temps, les yeux ouverts, selon la formule du coureur de bois, où l'indépendance était dans la nature de la nature 41 . Puisque le présent historique n'est qu'un hiatus, à cheval sur la «noirceur» qui a succédé à la «chute» et l'espoir d'un futur plus transparent, les projets d'avenir sont puisés dans les indices, les traces qu'a pu laisser dans la mémoire individuelle et collective ce «naturel du pays»: Une souvenance dla sorte de ceuses qu'on se souvient pareil même quand on s'en rappelle pus... St'un oubli qu'on se souvient...42

Ce «testament», cet héritage, inscrit dans la nature et dans l'inconscient individuel et collectif, n'a donc jamais été écrit - y manquait dmots dans lcode pour départager l'usufruit 43 - et, par conséquent, n'a jamais pu être reconstitué que par fragments et résonances, éparpillés tout au long de l'histoire. Comme nous avons essayé de le montrer plus haut, l'appropriation par la remise à l'endroit de l'histoire se fait, chez Germain, surtout en fonction du langage, de la lecture des signes. Dans ce sens, le déchiffrement de l'origine du récit historicofolklorique devient le récit des origines. Mais le sentiment d'une perte irrémédiable de la correspondance entre les mots et les choses, entre l'abstrait du langage codé et le concret du pays en tant qu'espace-temps de l'immédiat, de la parole non déguisée, l'a amené à prendre du recul vis-à-vis de sa propre réalité:

En 1960, on assiste à une tentative de retour à la nature ce qui ferme le cercle avec le régime français, deux cents ans plus tard. Mais ici, tout se trouve sur un plan abstrait. L'homme est redevenu marginal comme le coureur de bois du début à la différence qu'il nage dans le vague 44 .

En fait, même si dans sa démarche Germain n'échappe pas à une certaine fixation sur un passé devenu mythique, son entreprise de nature spéculaire vise plutôt le futur. Sa pratique l'a amené justement à ne pas oublier l'épaisseur du miroir, ni le rétrécissemnt engendré par l'emprise du simulacre.

\section{3. «L'ici-maintenant»: la problématique du miroir}

«L'ici-maintenant» est constitué chez Germain par l'espace-temps de la représentation: une brèche dans le temps indifférencié d'un pays sous le boisseau 45 . Toute l'alchimie du spectacle consiste alors à créer le moment propice à l'inauguration d'un devenir (le rêve commun) par la transfiguration du présent. Sous la forme de figures allégoriques qui s'enchâssent et s'ouvrent vers l'ensemble de la question générale de la québécité, la scène du défilé de la Saint-

Montréal, VLB éditeur, 1979, p. 36-37.

41 Un pays dont la devise est je m'oublie, p. 15.

42 Ibid., p. 16.

43 Ibid.

44 Adrien Gruslin, «la Courtepointe de Jean-Claude Germain», le Devoir, 28 octobre 1978, p. 23.

45 Jean-Claude Germain, «Mémoire théâtrale», Jeu, 13, automne 1979, p. 15. 
Jean-Baptiste, racontée par Épisode Surprenant, en est l'expression la plus éloquente:

Monté sus mon char allégorique, avec mon chapeau d plumes d'une main pis mon épée dans l'autte... chtais lQUÉ-BEC... TOUTTE LE QUÉBECQUE! (...) Ouais! Toutte le Québec... en un seul É-p-i-s-o-d-e $S-u-r-p-r-e-n-a-n-t ! 46$

Point focal où convergent tous les regards et, partant, les directions de la perspective, le char allégorique devient le centre, le lieu de la recomposition d'un collectif par la recomposition d'un langage où la communauté se reconnaît - le partage, cher au dramaturge, des images culturelles «populaires». Dans ce sens, le char allégorique nous renvoie à son homologue, la scène de thêâtre qui à son tour réfléchit, toujours sur le mode allégorique, la société - dans ce cas le Québec, le pays tout entier. Dans la figure du char il y a celle de l'acteur/héros romantique, mais aussi celle de l'acteur/pays, celui qui incarne toutte la parade et, partant, tout le Québec. L'acteur renvoie donc au thêatre et par là à la communauté, qui renvoie à son tour à une identité culturelle réfléchie et enrichie par la scène. La structure est donc circulaire et lorsqu'il manque un anneau à la chaîne, tout le système en est affecté. Chez Germain, l'anneau manquant est justement celui de la communauté, de l'identité culturelle: l'âme égarée de sa diva, devenue de ce fait l'indiva, une star à qui on a ôté une jambe et dont les interlocuteurs sont touttes manchots dla même main!

Par l'utilisation systématique de la réduplication réflexive, Germain a fait de ce handicap le souci particulier d'un thêâtre dont la fonction est, d'après lui, celle de traduire une société à elle-même 47 . D'où les deux modes structurels de réduplication (thêâtre dans le théâtre, enchâssement), les figures réflexives de toutes sortes (images, chansons), mais aussi les objets/emblèmes comme la table des Sansoucis, la malle-armoire des comédiens ambulants, le téléphọe de la diva, etc.

Le meilleur exemple de thêâtre dans le thêatre est celui du procès des Sansoucis (Si les Sansoucis [...]). Mais la présence des musiciens dans les spectacles de la diva, ainsi que des trois frères dans A canadian play/Une plaie canadienne obéit au même principe de réduplication du rôle du public. Á ce procédé s'ajoutent les enchâssements de tout ordre, depuis les apartés et les anecdotes jusqu'au rêve dans le rêve, en passant par tous les prologues et épilogues directement adressés au public. À cause du sujet même de la pièce, les enchâssements foisonnent dans l'École des rêves. Ce procédé apparaît dans toutes les créations de Germain. L'effet le plus important qui se dégage de l'utilisation de ces deux mécanismes est celui de la polarisation de l'espace scénique, soit vers le centre de la scène - le thêâtre dans le thêâtre - soit vers un espace

46 Jean-Claude Germain, l'École des rêves, Montréal, VLB éditeur, 1979, p. 21. Souligné par l'auteur.

47 Jean-Claude Germain, «Mémoire théâtrale», Jeu, 13, automne 1979, p. 14. 
ouvert, comprenant la salle et la scène, les chansons et toutes les formes de discours directement adressés aux spectateurs. Cette polarisation entraine donc une sorte de fluctuation des limites de la scène, c'est-à-dire de la ligne qui sépare l'espace dramatique de l'espace de sa contemplation. On note même chez Germain une tendance à renverser ces deux espaces, et cela depuis Diguidi diguidi ha! ha! ha!

En fait, en l'absence d'un champ commun où puisse s'effectuer la communion intersubjective - ce sens québécois qu'il fallait donner au Québec - les inversions se multiplient à tous les niveaux et débouchent souvent sur une spécularité généralisée. La plus radicale et qui engendre toutes les autres, est celle de la réalité et de l'illusoire. La salle/communauté, n'ayant plus la clef du mystère, renvoie à la scène/allégorie du pays l'image réfléchie ou pervertie d'une représentation devenue pur jeu (de mots et de rôles), plaisir gratuit et sans postérité, l'apogée de l'acteur et de l'illusion. Car la vie est dans l'illusion du thêâtre et dans le rêve, la réalité devient fiction, cauchemar. Les acteurs de thêâtre retrouvent leur vérité dans le personnage (Épisode/Cyrano) et jouent ainsi leur propre rôle, conscients de leur statut d'acteurs et de leur rôle dans le théâtre du monde, dont ils sont aussi les spectateurs privilégiés:

Moué toute sque chsais çé quchus un acteur! Pis quand la pièsse est drôle, chus drôle! Pis quand est trisse, chus trisse! (...) eu-z-auttes... y sont comme Hermogène pis Villaminé... y savent pas qu'y sont dé-z-acteurs! 48

Au niveau du travail théâtral, la salle devient un élément de décor, une illusion de plus, comme dans le rêve d'Épisode Surprenant. Le spectateur devient de ce fait un voyeur et le symbole s'abolit dans l'ambiguîté irréductible du jeu des signes. L'éclipse de l'auteur en tant que centre et lieu de regroupement des significations - le maître des situations dramatiques et des mots - s'accomplit dans et par le fonctionnement de son propre discours. Ainsi, la diva - un «intellectuel» qui réfléchit dans une langue populaire pour ne pas être ridicule 49 - se dérobe plus qu'elle ne se dévoile dans le vertige de la métamorphose et du dédoublement (les Nuits de l'Indiva). Il ne lui reste que le rire, l'ivresse et le silence comme seuls moyens de se montrer (par opposition à se dire), et partant, maîtriser l'angoisse.

Mais comme un acteur sans le personnage n'intéresse parsonne, selon l'expression du comédien ambulant, il y a chez Germain un mouvement inverse, un essai d'intégrer la salle à l'espace dramatique, de mettre en scène le collectif devenu acteur. Avec la réflexion des comédiens ambulants sur leur propre métier (Shakespeare et Hugo ne sont pas loin), cette inversion de la perspective est exploitée dans toutes ses implications: Épisode! 25,000 parsonnes cé trop! (...) Cé

48 L'École des rêves, p. 48.

49 Jean-Claude Germain, «Personnages de femmes», Jeu, 13, automne 1979. p. 43-44. 
moué qui fait lChrisse pis j'ai l'impression dfaire dla figuration! 50

La foule protagoniste du spectacle reste pourtant une virtualité dans «l'icimaintenant» ou, comme le veut le dramaturge, un sou-ve-nir du fu-tur en e-x-i-l dans lpré-sent! 51

L'idée d'incarner l'obstacle pour mieux l'abattre -il faut tuer l'Anglais ${ }^{52}$ a débouché cependant sur l'artifice: le sacrifice du «Bonhomme» n'a pas comblé le vide laissé par la perte irrémédiable des origines: le puits/tombeau autour duquel évoluent les personnages dans A canadian play/Une plaie canadienne et, de la même façon, le téléphone de la diva sont les symboles d'une communication impossible.

En fait, au présent, il ne reste que la passion, la folie dérisoire ou le désespoir des gens du théâtre et la volupté du souvenir. Moins pragmatique que ses pairs du théâtre de «la petite époque», la diva dézâmée, protagoniste de ce théâtre du présent, porte les marques d'une révolte et d'un certain désespoir qui ne sont pas étrangers à Germain lui-même: Pendant qu'moué, jme dézâme à leu dire sque ch'sens pis sque ch'pense vraiment! Pas d'danger que j'lé fasse mourir d'apoplexie...53

Sa décision de passer à l'Histoire, de terminer, une fois pour toutes avec cette période creuse 54 , n'établit pas pour autant une rupture, du moins au niveau de son discours didactique. Pris au piège d'une machinerie conceptuelle fondée sur la négation de toute altérité, Germain insiste sur la nécessité de trouver un raccourci pour faire coïncider le discours culturel québécois, c'est-à-dire le discours des origines, avec sa perception du monde moderne ${ }^{55}$, c'est-à-dire la réalité obsédante de l'aléatoire de l'existence - le sens de la vie. Point56.

En attendant que l'étape $d u$ choix soit entamée, il faudrait peut-être remettre à l'endroit une de ses formules: la question n'est plus de trouver des réponses mais de savoir qui pose les questions 57 .

50 Un pays dont la devise est je m'oublie, p. 105.

51 Les Nuits de l'Indiva, p. 153.

52 Jean-Claude Germain, «Un pouvoir par la langue», Jeu, 13, automne 1979 , p. 40.

53 Les Hauts et les bas d'la vie d'une diva: Sarah Ménard par euxmêmes, p. 68.

54 Jean-Claude Germain, «Mémoire théâtrale», Jeu, 13, automne 1979, p. 15.

55 Jean-Claude Germain, «la Culture aux prises avec le patrimoine», Jeu, 13, automne 1979, p. 42.

56 Jean-Claude Germain, «Un pouvoir par la langue», Jeu, 13, automne 1979, p. 40.

57 La question n'est plus de savoir qui pose les questions mais de trouver des réponses. (Ibid.). 